\title{
DIE DUALITAT ZWISCHEN TRADITIONELLEM AFRIKANISCHEN RECHT UND REZIPIERTEM EUROPÄISCHEN RECHT
}

\author{
Entwicklung und Problematik
}

\section{Begriffliche und zeitliche Abgrenzung}

Unter Dualität zwischen afrikanischem Recht und rezipiertem europäischen Recht versteht man das Nebeneinander zweier völlig verschiedener Rechtssysteme, das in Afrika durch das Erscheinen der Europäer entstanden ist. Abgrenzen möchte ich jedoch den zeitlichen Rahmen meiner Ausführungen in der Weise, daß ich die Betrachtung in dem Zeitpunkt beginnen lasse, in dem die afrikanischen Gebiete mehr oder minder stark unter den direkten Einfluß der großen Kolonialmächte geraten waren; dies bedeutet, daß die Zeit unberücksichtigt bleiben muß, in der lediglich Teile der später als Kolonien bekanntgewordenen Territorien, meistens ihre Küstenstriche, von Europäern besetzt waren. Der Schwerpunkt der Untersuchung erstreckt sich daher auf die Zeit nach der Berliner Kongo-Konferenz von $1884 / 1885$.

Nach dieser zeitlichen Abgrenzung ist als erstes die Frage zu klären, wie es überhaupt zur Dualität von afrikanischem und europäischem Recht kommen konnte. An dieser Stelle muß bereits eine Unterscheidung getroffen werden zwischen den ehemals britischen Gebieten und den Kolonien der anderen europäischen Mächte, in der Hauptsache also Frankreich, Belgien und Portugal. Bei allen europäischen Staaten war zwar gemeinsam die Rechtsauffassung vorhanden, daß ihre Staatsangehörigen ihr nationales Recht mit sich führen, wohin immer sie auch gehen mochten. Doch während es zu einem Prinzip des englischen Rechts gehörte, ein in einem fremden Land vorgefundenes Rechtssystem anzuerkennen, soweit es nach englischer Auffassung "reif" war, waren die anderen Mächte von der Überlegenheit ihrer Zivilisation und damit auch ihres Rechts gegenüber anderen Rechtsordnungen überzeugt und haben daher versucht, ihre Rechtsordnung an die Stelle des Rechts zu setzen, auf das sie stießen. Während die Engländer durch ihr Verwaltungssystem der „indirect rule“ die bereits vorhandenen Institutionen bewußt weiterbestehen ließen und somit auch dem afrikanischen Recht seinen Platz im Gesamtbereich einer Kolonie freiwillig einräumten, mußten die anderen Kolonialmächte im Laufe der Zeit erkennen, daß sich das afrikanische Recht nicht einfach ersetzen ließ, und daher gezwungenermaßen seine Weiterexistenz akzeptieren.

Bevor im einzelnen untersucht wird, wie sich die verschiedenen Kolonialmächte Europas dem afrikanischen Recht gegenüber verhalten haben, muß jedoch der Versuch gemacht werden, einige Wesensmerkmale des afrikanischen Rechts herauszuarbeiten, um überhaupt verstehen zu können, welchem Gebilde sich die Europäer in Afrika konfrontiert sahen. Vorausschicken möchte ich, daß es unmöglich ist, eine allgemein gültige Definition des afrikanischen Rechts zu finden. Schon die Verwendung des Begriffs "afrikanisches Recht" im Singular ist unkorrekt, da es e in afrikanisches Recht nicht gibt; es wäre daher richtiger, stets von afrikanischen Rechten zu sprechen, ganz korrekt ist eigentlich nur die Verwendung der Begriffe Stammesrechte, da der Ausdruck „afrikanisch“ im Zusammenhang mit Recht zu der Deutung Anlaß geben könnte, als handle es sich um ein dem ganzen Kontinent eigenes Grundsystem. Wenn im folgenden dennoch der Begriff „afrikanisches Recht" benutzt wird, so geschieht dies mit den soeben vorgebrachten Einschränkungen.

Das afrikanische Recht ist das Recht der verschiedenen Stämme Afrikas, das oft eng an authentische afrikanische Mythen anknüpft. Teilweise hat es sich durch den Einfluß des Islams zu einem Gemisch von Stammesrecht und islamischem Recht 
verbunden, wie z. B. in Mauretanien, Nordsenegal, Mali, Niger, Guinea, Tschad, Nordnigeria und Ostafrika, teilweise ist es auch durch das Christentum beeinflußt worden; dies geschah beispielsweise in Athiopien, wo bis 1960 die "Fetha Negast“, d.h. die "Gerechtigkeit der Könige“, eine Sammlung von Rechtsvorschriften, angewandt wurde, die auf das Konzil von Nicäa im Jahre $325 \mathrm{n}$. Chr. zurückgeführt werden und die von einem christlichen Ägypter im 13. Jahrhundert gesammelt und aufgeschrieben wurden. Sieht man von dem soeben zitierten Beispiel Äthiopiens ab und betrachtet man das in Madagaskar 1881 von der Königin Ranavalona II. erlassene, aus 305 Artikeln bestehende, schriftlich niederlegte Gesetz als zweite Ausnahme, so kann die allgemein gültige Behauptung aufgestellt werden, daß das afrikanische Recht ungeschrieben ist, d. h. auf oraler Überlieferung beruht, womit nicht ausgeschlossen ist, daß es sich um gesetztes Recht handelt, so daß die oft verwandte Bezeichnung "Gewohnheitsrecht" falsch ist. "Gewohnheitsrecht" bezieht sich auf den Geltungsgrund. Dieser ist im afrikanischen Recht nicht die Gewohnheit, sondern die Autorität eines wenn auch häufig mythisch verbrämten Gesetzgebers. Die Art der Rechtsüberlieferung ist keineswegs ein wesentliches Kriterium zur Unterscheidung des afrikanischen vom europäischen Recht. Ein sekundäres Kriterium zur Unterscheidung beider „Rechtssysteme“ ist auch, daß das afrikanische Recht sich fast immer ausschließlich auf die Regelung der Beziehungen der Mitglieder eines Stammes oder einer sonstigen Gemeinschaft untereinander bezog und Vorschriften für den Rechtsverkehr mit den Angehörigen anderer Gemeinschaften nicht kannte, oft einfach deshalb, weil ein Austausch mit Mitgliedern anderer Stämme nicht stattfand.

Das afrikanische Recht unterscheidet sich allerdings wesentlich in folgenden Punkten vom europäischen Recht: Im Fehlen einer Differenzierung zwischen Rechtssätzen und moralischen Verhaltensnormen, in der Verneinung der Macht des Menschen, Recht zu ändern, und in der Verneinung einer Auswirkung von Zeitabläufen auf bestehende Rechte. Für das afrikanische Recht ist der Mensch Sklave der Ordnung. Recht und Moral sind untrennbar verbunden mit ewigen Mythen oder mit einem Gesetz, das gegebenenfalls von Gott oder einer Gottheit gesetzt wurde. Die Furcht vor übernatürlichen Gewalten und vor der öffentlichen Meinung genügten im allgemeinen, um die Einhaltung der überlieferten Lebensformen zu sichern. Geboten fehlt daher oft die Verbindung mit Konsequenzen und Sanktionen, die sie nach europäischer Auffassung erst zu Rechtssätzen werden lassen, Recht und Moral sind nicht voneinander zu trennen. Dem afrikanischen Recht ist auch der Gedanke fremd, daß es der Mensch wagen dürfe, die Ordnung zu verändern. Selbst da, wo Normen von Stammesautoritäten gesetzt sind, geschieht dies nur, um ein und für alle Male klarzustellen, welche Vorschriften Geltung haben sollen und um zu vermeiden, daß die Ordnung durch veränderte Umstände oder auf Grund bestimmter Ereignisse abgewandelt werde. Im Gegensatz hierzu versteht sich das europäische Recht als ein Werk des Menschen. Er macht die Gesetze, die er benötigt, die jedoch nicht unabänderlich für alle Zeiten bestehen sollen, sondern die den veränderten Umständen angepaßt werden können. Der Mensch bewährt diese Rechtssätze mit Sanktionen und regelt auch ihren Vollzug, er sorgt für den Gehorsam. Das afrikanische Recht erhält seinen Gehorsam in starkem Maße durch außerrechtliche Kräfte, so daß man nur mit Vorbehalten überhaupt von afrikanischem $\mathrm{R}$ e $\mathrm{cht}$ sprechen kann, präziser wäre die Bezeichnung „afrikanische Ordnungsnormen “. Nach europäischer Vorstellung ist der Mensch Herr der Ordnung, für die Afrikaner ist er ihr Sklave oder Untergebener.

Ein weiterer wichtiger Unterschied besteht in der Rolle, die die Zeit bei der Ausgestaltung, Veränderung und Erneuerung des Rechts spielt. Nach europäischer Auffassung schafft die Zeit neue Situationen, die die Gegenwart von der Vergangenheit scheiden und die einen Fortschritt erlauben, was zur Folge hat, daß das Recht diesem Fortschritt angepaßt werden muß. Dem afrikanischen Recht ist der 
Begriff der fortschreitenden Zeit als relevantes Ereignis, das gegebenenfalls eine Änderung des Rechts bewirken kann, unbekannt. Zwei Beispiele werden diese Einstellung des afrikanischen Rechts verständlich machen: Den Vorstellungen des Afrikaners waren weder der Begriff der Rechtskraft der Entscheidung noch jener der Ersitzung bekannt. Beiden Instituten kommt nach europäischer Rechtsauffassung die Hauptaufgabe zu, dafür zu sorgen, daß umstrittene Rechtsverhältnisse nach Ablauf einer bestimmten Zeit im Fall der Ersitzung oder mit dem Eintritt eines bestimmten Ereignisses, der Rechtskraft des Urteils, endgültig geregelt werden. Hierbei nimmt es der europäische Gesetzgeber im Interesse des Rechtsfriedens in Kauf, daß jemand in eine Position versetzt wird, die ihm nicht zusteht, die ihm aber nicht mehr streitig gemacht werden kann, weil entweder der wahre Berechtigte während der Ersitzungszeit seine Ansprüche nicht geltend machte, so daß diese verfielen, oder weil den Ansprüchen die Rechtskraft gegenübersteht, selbst wenn ein Fehlurteil ergangen sein sollte. Eine solche Vorstellung, daß durch Zeitablauf neue rechtliche Situationen geschaffen werden können, ist dem afrikanischen Denken fremd. Diese Irrelevanz des Zeiteinflusses geht im afrikanischen Recht auch daraus hervor, daß es die Rolle des Individuums oder der Familie im engen europäischen Sinne für völlig unbeachtlich hielt und nur in Kategorien mehr oder minder großer Gemeinschaften wie Stämme, Kasten oder Dörfer dachte. Diese Großgemeinschaften sind im großen und ganzen von tiefgreifenden Erschütterungen oder Veränderungen, die das Recht modifizieren könnten, frei, sie haben ewigen Bestand, während im Leben eines einzelnen oder einer kleineren Gruppe von Menschen sehr schnell so wesentliche Veränderungen etwa durch Todesfälle eintreten können, daß auch das Recht der neuen Situation angepaßt werden muß.

Dieses war in großen Zügen die Situation, der sich der europäische Gesetzgeber in Afrika gegenübergestellt sah, der versuchen mußte, ein System $\mathrm{zu}$ finden, das Nebeneinander von zwei Menschengruppen rechtlich zu regeln, wovon die eine sich als unabänderlichen Rechtsbräuchen und -sitten unterworfen betrachtete, während die andere modernes Recht anzuwenden gewohnt war.

\section{Die Haltung der Kolonialmächte gegenüber dem afrikanischen Recht}

\section{Die Haltung Frankreichs}

Frankreich war von der Überlegenheit seines eigenen Rechts gegenüber dem afrikanischen Recht überzeugt und daher bemüht, seine Kolonien mit modernen Gesetzen auszustatten. Unterstützt wurde es durch die Missionare, die die Bewohner der afrikanischen Gebiete zum Christentum bekehren wollten und die daher ein Interesse daran hatten, daß insbesondere Sitten und Gebräuche, die mit den sittlichen und ethischen Ansprüchen des Christentums unvereinbar waren, nicht länger geduldet wurden. Der französische Gesetzgeber hielt es für seine vordringliche Aufgabe, die Afrikaner mit der "Wohltat" seiner Gesetze zu beglücken. Im Zuge der französischen Assimilationspolitik hatten alle frei Geborenen in den Kolonien bereits 1833 gleiche politische und zivile Rechte erhalten wie die Franzosen selbst. Es waren als erste die Richter, die im Laufe der Zeit feststellen mußten, daß sich die einheimische Bevölkerung den französischen Rechtsregeln nicht unterwarf, sondern es vorzog, weiter in ihrer angestammten Ordnung zu leben. Der Gesetzgeber war also gezwungen, sich der neuen Situation anzupassen. Zwischen den Jahren 1857 und 1910 wurden zunächst für Senegal, anschließend für alle anderen frankophonen Länder mit islamischen Bevölkerungsgruppen ein islamisches Statut, später für die nicht islamischen Eingeborenen in AOF und AEF ein Eingeborenenstatut erlassen, die es den Afrikanern erlaubten, entweder nach den Vorschriften des Korans oder nach ihrem Stammesrecht zu leben. Leicht abgeänderte Texte ergingen nach der Übernahme der Mandatsgebiete Togo und Kamerun für diese 
beiden Gebiete, während ein persönliches madagassisches Statut bereits bei der Annexion der Insel 1896 beibehalten worden war.

Bis 1946 ging die Unterscheidung zwischen "Statut Civil Français“ und „Statut Civil Local" Hand in Hand mit der Unterscheidung in Bürger (citoyens) und Untergebene (sujets). Bürger waren diejenigen, die dem französischen Recht in seiner Gesamtheit unterworfen waren, Nichtbürger dagegen jene Personen, die einem anderen Rechtssystem unterstanden. Durch die Verfassung von 1946 wurden alle Bewohner der französischen Kolonien „citoyens français“, doch betraf diese Neuregelung lediglich die Ausübung der Bürgerrechte. Zivilrechtlich bestand weiterhin der Unterschied zwischen "Citoyen de Statut Civil Français" und "Citoyen de Statut Local". Auf den lokalen Zivilrechtsstatus konnte in einem besonderen Verwaltungs- oder Gerichtsverfahren verzichtet werden, wobei der Antragsteller insbesondere nachweisen mußte, daß er sich der französischen Lebensart angepaßt hatte; er wurde sodann dem französischen Status unterstellt.

Eine weitere tiefgreifende Änderung hatte die Verfassung von 1946 insofern zur Folge, als alle Bewohner der französischen Kolonien, unabhängig von ihrem persönlichen Status, einheitlich dem 1946 verkündeten Strafgesetzbuch unterworfen wurden, das eine wenige Abweichungen enthaltende Version des Strafgesetzes der Metropole darstellte. Die bis 1946 auf dem Strafrechtssektor tätigen Eingeborenengerichte wurden aufgelöst; diese hatten Stammesrecht angewandt, das jedoch vom französischen Gesetzgeber von solchen Bräuchen befreit worden war, die mit französischen Rechtsvorstellungen unvereinbar waren.

Im Zivil- und Handelsrecht waren die dem „Statut Civil Local“ unterworfenen Afrikaner Eingeborenengerichten unterstellt. Diese Gerichte wurden jedoch von französischen Beamten geleitet. Afrikaner waren dort nur Beisitzer mit beratender Stimme. Durch den starken Einfluß der französischen Beamten auf die Prozeßführung wurde daher der Zivilprozeß sehr stark von französischen Verfahrensvorschriften durchdrungen. Aber auch das materielle Stammesrecht wurde durch die französische Gesetzgebung beeinflußt, beispielsweise durch die Festsetzung eines Mindestalters für die Eheschließung, durch Abhängigmachung der Gültigkeit der Ehe vom Einverständnis beider Ehegatten, durch die Einführung der Registrierung von Geburten, Eheschließungen und Todesfällen; der Brautpreis wurde für Volljährige oder Geschiedene, die wieder heiraten wollten, abgeschafft; im Vertragsrecht wurde die Möglichkeit geschaffen, Verträge von Verwaltungsbeamten bestätigen $\mathrm{zu}$ lassen, um eine größere Beweissicherheit zu gewährleisten; die Polygamie wurde zwar nicht offiziell abgeschafft, aber zur Durchsetzung der Einehe wurde vielfach den Ehepartnern die Verpflichtung auferlegt, erst dann eine neue Ehe einzugehen, wenn eine noch bestehende geschieden war.

Auf dem Gebiet der Gesetzgebung für die Kolonien bestand seit 1870 der allgemeine Rechtsgrundsatz der Spezialität; er besagte, daß vom französischen Parlament verabschiedete Gesetze in den Kolonien nur Anwendung fanden, wenn sid. dies aus dem Sinn und Zweck des Gesetzes selbst ergab (z. B. das Arbeitsgesetzbuch für Úbersee von 1952) oder wenn der Gesetzgeber die Absicht der Anwendbarkeit des Gesetzes für Übersee ausdrücklich kundtat. Daneben hatte die Exekutive die Befugnis, alle Fragen, die nicht durch ein Gesetz geregelt worden oder der Regelung durch ein Gesetz vorbehalten waren, durch Dekrete für die Kolonien zu regeln. $\mathrm{Da}$ das Parlament nur selten Gesetze für die Kolonien ausgearbeitet hat, war die Kompetenz der Exekutive so groß, daß man allgemein von der „Regelung duch Dekrete" sprach, wenn man die Gesetzgebung für die Kolonien charakterisieren wollte. Zwei Wege waren bei diesem Verfahren üblich: Entweder erließ die Regierung ein spezielles Dekret für die Kolonien, oder sie dehnte ein französisches Gesetz nach entsprechender Abänderung, durch die es den Verhältnissen in Úbersee angepaßt wurde, durch Dekret auf die Kolonien oder Teile von ihnen aus. 
Generell bestand für die Anwendbarkeit des afrikanischen Rechts die Einschränkung, daß es nur gültig war, wenn es nicht gegen den französischen „ordre public" verstieß, d.h. nicht gegen die guten Sitten und nicht gegen Sinn und Zweck des französischen Rechts.

\section{Die belgische Haltung}

Vor der Gründung des unabhängigen Kongostaates galt für jeden Stamm innerhalb des Territoriums, das später als belgischer Kongo bekannt wurde, ein eigenes Stammesrecht, das entweder auf den von der herrschenden Schicht der Eingeborenen erlassenen Vorschriften, auf Gerichtsurteilen oder auf Sitten und Bräuchen beruhte. Während der Zeit des unabhängigen Kongostaates von 1885-1908 galt dieses Prinzip, daß die Afrikaner ihrem Stammesrecht unterworfen bleiben, weiter. Es wurde durch die Kolonialcharta der belgischen Regierung ausdrücklich bestätigt. Zur Durchsetzung ihres Rechts konnten sich die Afrikaner entweder an europäische Gerichtshöfe wenden, die in solchen Fällen afrikanisches Recht anwandten, oder sie begaben sich vor die "Eingeborenengerichte“, die teilweise nur von Afrikanern, teilweise von Afrikanern unter Vorsitz eines Beamten der belgischen Verwaltung, besetzt waren.

Die Anwendung des Stammesrechts fand ihre Einschränkung, wenn es dem geschriebenen Recht oder dem „ordre public" widersprach. Zu solchen, nicht mehr anwendbaren Bräuchen gehörten $u$. a. die Eheschließung ohne Zustimmung beider Ehegatten, Ehescheidungen auf Wunsch der Familienangehörigen eines Ehepartners, willkürliche Machtausübung von Stammeshäuptlingen und sittenwidrige Verpflichtungen. Hierzu gehörten ferner alle mittels Hexerei erlangten Beweise in Gerichtsverfahren. Häufig wurden nicht mehr zulässige Sitten und Bräuche nicht formell verboten und unter Strafe gestellt, sondern lediglich für unanwendbar erklärt. Dies hatte zur Folge, daß jedes europäische Gericht von Amts wegen verpflichtet war, ein Urteil aufzuheben, das sich auf eine Sitte oder einen Brauch bezog, dessen Anwendung der Gesetzgebung oder dem „ordre public“ widersprach.

Die Kongolesen hatten seit 1952 die Wahl, sich aus dem Einflußbereich des afrikanischen Rechts zurückzuziehen, indem sie sich durch Immatrikulation der Regelung des Zivilgesetzbuchs unterwarfen. Diese Registrierung, die von einem gewissen Bildungsstand und einer bestimmten Lebensart abhängig war, mußte vor den höheren Gerichten beantragt werden. Wurde dem Antrag entsprochen, lebte der Antragsteller fortan unter einem Zivilgesetz, das speziell für den Kongo ausgearbeitet worden war und nicht identisch mit dem belgischen Zivilgesetzbuch war. Als Folge der Immatrikulation, die als solche lediglich zivilrechtliche Konsequenzen hatte, wurde der Afrikaner auf Grund mehrerer zusätzlicher Verordnungen weitgehend dem Europäer gleichgestellt: In Strafverfahren waren für ihn nur noch die ordentlichen Gerichte zuständig, im Bodenrecht konnte er individuelles Eigentum erwerben und ohne Beschränkungen darüber verfügen. Es fand also ein Assimilierungsprozeß mit den Europäern statt.

$\mathrm{Da}$ sich die belgische Verwaltung aber zunehmend Klarheit darüber verschaffte, daß sie durch das System der Immatrikulation zwei Klassen von Afrikanern schuf, die sich mehr und mehr entfremdeten, erfand sie eine zwischen beiden Fronten liegende dritte Lösung: Auf Antrag konnte interessierten Afrikanern die sogenannte „Carte du Mérite Civique" zuerkannt werden, durch die ihre Inhaber den Europäern nur teilweise gleichgestellt wurden, so daß sie stärker mit ihrem Stamm verbunden blieben. Bedeutung hatte die Zuerkennung der Karte besonders im Grundstücksrecht, wo die betreffenden Afrikaner von der Genehmigungspflicht für gewisse Geschäfte freigestellt waren. 


\section{Die portugiesische Haltung}

Grundsätzlich gilt für die portugiesischen Überseebesitzungen Guinea, Angola und Moçambique portugiesisches Recht. Auf den Gebieten des Familienrechts, des Erbrechts, des Vertragsrechts und des Eigentumsrechts findet jedoch afrikanisches Recht Anwendung. Dieses darf aber nicht berücksichtigt werden, wenn es gegen Prinzipien der Humanität oder gegen Grundvorstellungen der Moral verstößt oder wenn es die Ausübung der Souveränitätsrechte Portugals behindern würde.

Die Portugiesen gewähren den Afrikanern zwei Alternativen, sich dem Eingeborenenrecht zu entziehen. Entweder können diese beantragen, teilweise dem portugiesischen, teilweise dem afrikanischen Recht unterstellt $\mathrm{zu}$ werden; ist die Wahl getroffen, so ist sie endgültig; der betreffende Afrikaner untersteht dann für den von ihm gewählten Bereich ausschließlich dem portugiesischen Recht. Die zweite Alternative ist die völlige Unterwerfung unter portugiesisches Recht. Hierbei sind jedoch von der portugiesischen Verwaltung einige Bedingungen eingeführt worden, die der Antragsteller zu erfüllen hat: Er muß 18 Jahre alt sein, fließend die portugiesische Sprache beherrschen, einen Beruf ausüben, der ihn in die Lage versetzt, sich und seine Familie zu unterhalten, oder das zur Erfüllung dieser Aufgaben erforderliche Vermögen besitzen. Der Antragsteller darf sich ferner nicht dem Militärdienst entzogen haben, seine Führung muß tadellos gewesen sein und sein gesamter Lebensstil muß ihn als würdig ausweisen, alle Rechte eines portugiesischen Bürgers zu übernehmen.

Das Gerichtsverfahrensrecht regelt sich teilweise nach portugiesischem, teilweise nach afrikanischem Recht. Die allgemeinen Gerichte wenden portugiesisches Recht an, sogenannte Stadtgerichte sind für das afrikanische Recht zuständig. Aus der Tatsache, daß die allgemeinen Gerichte auch für Streitigkeiten zwischen Afrikanern und Europäern zuständig sind, könnte auf eine Benachteiligung der Afrikaner geschlossen werden. Die Härte dieser Bestimmung wird jedoch in zweierlei Weise gemildert: Einerseits verläuft in solchen Fällen das Verfahren in einer Weise, die dem vor den Stadtgerichten angeglichen ist, weil man der Meinung ist, daß hierdurch die Afrikaner am besten in die Lage versetzt werden, den Ausführungen folgen zu können; andererseits haben die Richter Anweisung, das portugiesische Recht nicht streng nach den Buchstaben anzuwenden sondern insbesondere $\mathrm{zu}$ berücksichtigen, wieweit der Afrikaner die nach portugiesischem Recht eintretenden Rechtsfolgen vorhersehen konnte; bei dieser Beurteilung sollen insbesondere der Charakter und die Mentalität der betreffenden afrikanischen Prozeßpartei gewürdigt werden.

\section{Die Haltung Englands gegenüber dem afrikanischen Recht}

Das englische Parlament hatte die Gesetzgebungsgewalt nur für seine Staatsangehörigen, d. h. somit nur für das Mutterland und die Kolonien, deren Bewohner „British Subjects" waren, aber auf indirektem Wege wurde die Gesetzgebung durch die Foreign Jurisdiction Acts auf die anderen territorialen Gebilde ausgedehnt, so daß für unsere Betrachtung die Unterschiede zwischen Colony, Protectorate, Protected State und Trust Territory unwichtig sind.

Gesetze Englands wurden in der Regel durch einen besonderen Gesetzgebungsakt, üblicherweise in der Form einer "Order in Council“ auf das jeweilige Kolonialgebiet ausgedehnt. Hierbei handelte es sich um die Vorschriften von allgemeiner Bedeutung, während weniger wichtige Gesetze von den gesetzgebenden Körperschaften in den afrikanischen Territorien, den "Legislative Concils“, ausgearbeitet und vom Gouverneur verkündet wurden.

Der allgemein beschrittene Weg für die Ausdehnung des englischen Rechts auf die afrikanischen Gebiete war folgender: Durch eine königliche „Order in Council“ 
wurde bestimmt, daß von einem bestimmten Tage an die Gesetze von allgemeiner Anwendbarkeit sowie die Entscheidungen des Common Law und der Equity, wie sie zu diesem Zeitpunkt in England in Kraft waren, für ein bestimmtes afrikanisches Territorium geltendes Recht sein sollten; diese Zeitpunkte der sogenannten allgemeinen Rezeption des englischen Rechts waren z. B.: für Sierra Leone das Jahr 1862, für Ghana 1874, für Gambia 1888, für Kenia 1897, für Nigeria 1900, für Malawi und Uganda 1902, für Sambia 1911, für Tanganjika 1920. Daneben gab es die partielle Rezeption, die darin bestand, daß die afrikanischen Territorien solche britischen Vorschriften übernahmen, die nach dem Datum der allgemeinen Rezeption in England erlassen worden waren. Zu erwähnen ist hier z. B. das Gesellschaftsrecht, das die abhängigen Gebiete von England übernahmen und teilweise sogar jeweils dem Stand der großen Revisionen in England in den Jahren 1929 und 1948 angepaßt haben.

Die Normen des geschriebenen englischen Rechts wurden den Umständen angepaßt und mit Änderungen versehen, die der besonderen Situation des betreffenden afrikanischen Territoriums Rechnung trugen. Für die lokalen Gesetzgebungskörperschaften bestand ebenso wie für die Richter die Möglichkeit, aus eigener Initiative Normen des englischen Rechts auszuschließen oder zu verändern. Bestimmte Materien jedoch waren der ausschließlichen Zuständigkeit des britischen Parlaments vorbehalten.

Schwierige Probleme warf die Beurteilung der Frage auf, welche Entscheidungen englischer Gerichte auf die Kolonialgebiete Anwendung fanden. Unbestritten war, daß solche Gerichtsentscheidungen bindend waren, die in England vor dem Datum der allgemeinen Rezeption des englischen Rechts durch ein afrikanisches Territorium ergangen waren. Hierbei hat sich sogar eine Praxis durchgesetzt, die der grundsätzlichen Regelung von der bindenden Kraft des Präjudizes im englischen Recht widersprach: Ublicherweise besagte diese Theorie, daß durch ein von einem Gericht gefälltes Urteil dieses Gericht selbst sowie alle ihm nachgeordneten Gerichte gebunden waren. Bei Gerichtsentscheidungen englischer Gerichte, die vor dem allgemeinen Rezeptionsdatum ergangen waren, war jedoch zu beobachten, daß sich auch höhere Kolonialgerichte an Urteile gebunden fühlten, die ein ihnen untergeordnetes englisches Gericht gesprochen hatte. Dagegen waren Urteile, die nach der Rezeption ergangen waren, lediglich von "höchster Überzeugungskraft" für die Kolonialgerichte, hatten aber keine bindende Wirkung. Nicht bindend waren außerdem Urteile, die Rechtssätze betrafen, die nur auf das englische Mutterland Anwendung fanden. Schließlich wurden Rechtssätze des Common Law oder der Equity durch Gesetzgebungsakte aufgehoben, die das Richterrecht abänderten. Dieser Grundsatz, daß ein Gesetz entgegengesetztes Richterrecht aufhebt, wurde auch auf solche Normen angewandt, die von den Gesetzgebungskörperschaften eines Kolonialgebietes erlassen worden waren.

Die englische Kolonialverwaltung ließ, wie bereits erwähnt, die nach afrikanischen Bräuchen bestehenden Einrichtungen weitgehend unangetastet. Das afrikanische Recht galt daher mit der Maßgabe weiter, daß es nicht gegen die Gerechtigkeit, Billigkeit oder das gute Gewissen verstoßen und nicht mit einem bestehenden englischen Gesetz unvereinbar sein durfte. An dieser Stelle kam auch in der britischen Auffassung der Gedanke der Uberlegenheit des geschriebenen Rechts gegenüber dem nur mündlich überlieferten Stammesrecht zum Durchbruch.

Neben den für die Europäer zuständigen ordentlichen Gerichten, deren Hierarchie dem englischen Gerichtsaufbau entsprach, waren Eingeborenengerichte für Rechtsfragen zuständig, an denen nur Afrikaner beteiligt waren: ihre Zuständigkeit beschränkte sich nicht nur auf Afrikaner, die aus dem betreffenden Territorium stammten, sondern auch auf Afrikaner aus anderen Gebieten, die nur zeitweilig der Jurisdiktionsgewalt des betreffenden Gerichts unterstanden, sofern „ihre allgemeine Lebensart derjenigen anderer Eingeborenengemeinschaften entsprach". Neben den erstinstanzlichen Eingeborenengerichten gab es zum Teil auch Beru- 
fungsgerichte für Eingeborene. Weitere Rechtsmittel gegen Entscheidungen eines Eingeborenen-Berufungsgerichts gingen dann jedoch an die Kolonialgerichte. Höchste Gerichtsinstanz war für alle Kolonialgebiete der Privy Council der Königin in London. Bemerkenswert ist ferner, daß es regional zuständige Berufungsgerichte gab, die für mehrere Territorien jeweils das nach dem Privy Council höchste Gericht darstellten: Der West African Court of Appeal war zuständig für Ghana, Gambia, Sierra Leone und Nigeria, der East African Court of Appeal für Kenia, Uganda und Tanganjika, der Court of Appeal for Rhodesia and Nyasaland für Nord- und Südrhodesien und Njassaland. Die schwierige Frage, inwieweit die Urteile eines Kolonialgerichts die Gerichte anderer Kolonialgebiete banden, kann im Rahmen dieser Untersuchung nicht geklärt werden.

Bestimmte Rechtsgebiete, wie z. B. das Eherecht, das Familienstandsrecht, das Erbrecht und das Sorgerecht für Kinder sowie das Grund- und Bodenrecht nach Vorstellungen des afrikanischen Rechts, wurden ausschließlich von Eingeborenengerichten entschieden. Nur in Ausnahmefällen konnte der Gouverneur die Berufung an ein Kolonialgericht zulassen. Diese Berufungsmöglichkeit darf nicht verwechselt werden mit der den britischen Beamten zustehenden generellen Befugnis, alle Urteile der Eingeborenengerichte daraufhin zu überprüfen, ob sie der Gerechtigkeit, den guten Sitten, dem Gewissen oder einem geschriebenen Gesetz widersprachen. Von der Regel, daß afrikanisches Recht nur auf die Regelung der Beziehungen der Afrikaner untereinander anzuwenden war, gab es insofern eine bedeutende Ausnahme, als in Fällen, in denen sich Afrikaner und Nichtafrikaner als Parteien gegenüberstanden, afrikanisches Recht angewandt werden konnte, falls die Anwendung rein englischen Rechts zu einer untragbaren Ungerechtigkeit gegenüber einer der Parteien geführt hätte.

Im Zuge der Entwicklung wurde den Eingeborenengerichten auf Einzelgebieten die Rechtsprechungsgewalt auch für Zivil- und Strafsachen zuerkannt, die nach geschriebenem Recht geregelt waren: Sie wurden z. B. in Zivilverfahren für Streitgegenstände bis zu einem bestimmten Höchstbetrag, in Strafprozessen bei Vergehen bis zu einer festgelegten Höchststrafe, die die Todesstrafe sein konnte, zuständig, aber beschränkt auf solche Fälle, in denen Afrikaner Parteien oder Angeklagte waren.

Im Gegensatz zu den Bestimmungen bei den Franzosen, Portugiesen und Belgiern sah das britische Kolonialsystem keine Möglichkeit vor, daß ein dem afrikanischen Recht unterworfener Afrikaner auf seinen Status verzichten und den eines Europäers erlangen konnte. Lediglich in einem Gerichtsverfahren konnte ein Afrikaner Einzelbereichen des europäischen Rechts unterworfen werden. Hierbei entwickelte sich folgende Gerichtspraxis: Keine Partei ist berechtigt, sich auf afrikanisches Recht oder Brauchtum zu berufen, wenn sich aus einem ausdrücklichen Vertrag oder aus der Natur des Rechtsgeschäfts ergibt, daß diese Partei damit einverstanden war, daß ihre aus dem Rechtsgeschäft herrührenden Verpflichtungen ausschließlich durch englisches Recht geregelt werden sollen oder daß derartige Rechtsgeschäfte dem afrikanischen Recht unbekannt sind.

\section{Gemeinsame Ergebnisse aus der Kolonialzeit}

Bevor ich mich der Haltung der afrikanischen Regierungen gegenüber dem Dualismus zwischen afrikanischem und europäischem Recht zuwende, die diese nach Erlangung der Unabhängigkeit eingenommen haben, sollen noch einmal kurz die wesentlichen Ergebnisse zusammengestellt werden, die in dem Augenblick vorliegen, als die Europäer sich anschicken, als Kolonialmächte abzutreten und den Afrikanern die volle Souveränität zu übertragen.

a) Das europäische Recht hat sich in allen Bereichen durchgesetzt, in denen die Europäer praktisch alleine tätig waren, wie z. B. im Handel, im Bergbau, in der Industrie. 
b) Es hat sich ferner da behauptet, wo das afrikanische Recht lückenhaft war oder sich nicht an die neuen veränderten Umstände anpassen konnte, wie dies z. B. überall dort geschah, wo plötzlich der Rechtsverkehr zwischen Angehörigen verschiedener Gemeinschaften geregelt werden mußte; hierzu gehörten etwa das Arbeitsrecht oder aber die Probleme, die das Wohnen und Leben in Städten mit sich brachten.

c) Das afrikanische Recht dagegen überlebte in den Bereichen, deren Regelung die Kolonialmächte bewußt nicht in eigene Regie übernommen hatten, sondern wo sie bestehenden Institutionen ihren Raum ließen, sieht man von solchen Bräuchen $\mathrm{ab}$, die nicht aufrecht erhalten werden konnten, da sie Grundsätzen des europäischen Rechtsdenkens widersprachen. $\mathrm{Zu}$ diesen Gebieten gehörte das Eherecht, das Familienrecht, das Erbrecht sowie das Grund- und Bodenrecht.

Zieht man eine Schlußbilanz, so gelangt man zu der Feststellung, daß das afrikanische Recht in den meisten Fällen vom rezipierten Recht verdrängt worden ist. Es fehlte ihm die Kraft zur Anpassung an völlig veränderte Verhältnisse. Dazu kam, daß die Europäer nicht geneigt waren, einen positiven Beitrag zur Erhaltung und Fortentwicklung des afrikanischen Rechts zu leisten. Im Grunde waren die Kolonialmächte damit zufrieden, wenn sie sich um bestimmte Lebensbereiche der Afrikaner, und somit auch um die hiermit zusammenhängenden Rechtsfragen, nicht zu kümmern brauchten.

\section{Die Haltung der afrikanischen Regierungen nach der Unabhängigkeit gegen- über dem afrikanischen Recht}

Mit einer gewissen Spannung mußte man die Haltung der Afrikaner bei Erlangung der Unabhängigkeit gegenüber ihrem eigenen traditionellen Recht beobachten. Doch sollte es sich bald zeigen, daß im Zuge des sich nun frei entfaltenden afrikanischen Nationalismus eine Rückkehr zum afrikanischen Recht und eine radikale Abkehr vom Rechtsdenken der Kolonialmacht nicht eintraten. Für die Afrikaner stellte sich als Hauptproblem die Aufgabe, geeignete Mittel und Wege zu finden, den von der Kolonialmacht übernommenen Staat in einer Weise weiterzuführen, daß er am modernen zwischenstaatlichen Verkehr als gleichberechtigtes Mitglied teilnehmen konnte. Ausnahmslos alle afrikanischen Regierungen haben erkannt, daß sich dieses Unternehmen nur mit modernen Gesetzen zum Erfolg führen läßt und daß das afrikanische Recht zur Bewerkstelligung dieser Aufgaben denkbar ungeeignet ist. Daher ist kein afrikanischer Staat dazu übergegangen, europäisches Recht in größerem Umfang zu beseitigen. Es ist eher die Tendenz zu erkennen, daß das afrikanische Recht durch die Unabhängigkeit der afrikanischen Staaten stärker bedrängt worden ist als zur Zeit der europäischen Kolonialherrschaft. Folgende Beispiele sollen diese Feststellung unterstreichen:

1. Verfassungs recht: Verdrängt wurden große Teile des afrikanischen Rechts dadurch, daß sich alle afrikanischen Länder eine Verfassung zulegten: Die Religionsgebundenheit des traditionellen Rechts wurde dadurch beseitigt, daß sich die meisten Staaten als „weltlich“ proklamierten. Der afrikanische Familienbegriff, beruhend auf den Vorstellungen der Großfamilie oder der stammesmäßig gebundenen Einheit, wurde durch den europäischen Familienbegriff ersetzt. Zahlreiche Verfassungen schützen ausdrücklich Ehe und Familie im europäischen Sinne. Auch die Verankerung des Grundrechts Eigentum in vielen Verfassungen betrifft den Grundeigentumsbegriff moderner Prägung, nicht den im afrikanischen Recht vorherrschenden des Kollektiveigentums.

2. Verfahrensrecht: Auf diesem Gebiet wurden weitgehend europäische Verfahrensordnungen als nationale Gesetze in den verschiedenen Staaten übernommen. Hier werden sich die Afrikaner nach den Vorstellungen ihrer Gesetzgeber an Auffassungen gewöhnen müssen, die den ihrigen völlig entgegengesetzt sind. 
Das europäische Recht wird von dem Gedanken beherrscht, daß es Aufgabe des Richters ist, jedem das Seine zuzuerkennen; durch das Urteil soll ein Fall endgültig entschieden werden, in dem ein im voraus konzipiertes Gesetz auf den Einzelfall angewendet wird; der Richter ist daher nach europäischer Auffassung ein spezialisierter Techniker, der das Recht zu kennen hat, es interpretiert und anwendet. Die Auffassung des traditionellen afrikanischen Rechts läuft dagegen darauf hinaus, $\mathrm{daß}$ es erstes Ziel eines Prozesses zu sein hat, die Parteien durch Meinungsaustausch in Form des Palavers wieder zu versöhnen. Urteilen beinhaltet daher für den Afrikaner nicht Anwendung eines Gesetzes auf einen bestimmten Fall, sondern behutsame Annäherung der verfeindeten Parteien mit dem Ziel, wieder ein gutes Einvernehmen zwischen beiden herzustellen, der Begriff des Vergleichs im europäischen Recht ist hier eher am Platze.

3. Strafrecht: Auch auf diesem Gebiet macht sich die Tendenz bemerkbar, das afrikanische Recht immer mehr zu verdrängen. Hierbei darf aber nicht übersehen werden, daß es wahrscheinlich keinen Bereich gibt, in dem eine einheitliche Gesetzgebung aus grundsätzlichen Erwägungen notwendiger zu sein scheint als im Strafrecht. Ein Staat kann es sich nicht leisten, in seinem Hoheitsgebiet mehrere Strafrechtsysteme zu dulden, die darüber hinaus noch größtenteils auf ungeschriebenem, oft unsicherem Recht beruhen. Die Rechtssicherheit könnte unter diesen Umständen nicht gewährleistet werden. Daneben wäre es fraglich, wie in solchen Fällen der teils in den Verfassungen, teils in den Strafgesetzen verankerte Grundsatz des „nulla poena sine lege“ verwirklicht werden könnte, wenn kein Gesetz, sondern nur eine Überlieferung vorhanden ist. Die Beseitigung traditioneller Vorstellungen wird jedoch auf diesem Gebiet ebenfalls einen langwierigen Umstellungsprozeß der Afrikaner erfordern. Denn diese gehen bei der Frage nach dem grundsätzlichen Sinn der Strafauffassung von völlig anderen Voraussetzungen aus als der europäische Gesetzgeber. Das europäische Recht steht auf dem Standpunkt, daß durch einen Rechtsbruch die staatliche Ordnung gestört wird und daß dieser Staat nunmehr einen Anspruch gegen den Rechtsbrecher besitzt, ihn wegen seiner Tat zur Rechenschaft zu ziehen. Die afrikanische Auffassung beruht dagegen weitgehend auf dem Gedanken, daß der infolge einer Rechtsverletzung eingetretene Schaden wiedergutgemacht werden müsse. Der Gedanke, daß durch ein Verbrechen oder Vergehen die Rechtsordnung des Staates gestört und damit dieser Staat selbst verletzt worden sei, war dem Afrikaner fremd. Ihm ging es lediglich darum, in einem Strafverfahren die Schuld des Angeklagten festzustellen und dann Möglichkeiten zu finden, in welcher Weise der Verletzte oder Geschädigte so zu entschädigen sei, daß er nicht schlechter stehe als vor der Untat. Daher waren den Afrikanern Einrichtungen wie Gefängnisse oder Zuchthäuser weitgehend unbekannt, bevor die Europäer erschienen. Zwar kannten die Afrikaner auch die Möglichkeit, einen Übeltäter solange irgendwo festzuhalten, bis seine Verwandten oder Stammesangehörigen ihn durch Leistung an den Verletzten praktisch freikauften, doch hatte diese Einsperrung keinereli ehrenrührigen Charakter, sondern war lediglich eine Beugemaßnahme, um die Familie, die auf die Arbeitskraft des Täters angewiesen war, zur Leistung zu zwingen. Ebensowenig war dem Afrikaner ein staatlicher Henker bekannt; wenn eine Todesstrafe ausgesprochen worden war, so wurde es gewöhnlich den Verwandten oder Stammesangehörigen des Opfers überlassen, in welcher Weise sie den Mörder dem Tode zuführten.

Andererseits gibt es aber auch zahlreiche Beispiele dafür, daß sich afrikanische Rechtsbräuche erhalten ließen und in moderne Gesetze europäischen Vorbilds eingearbeitet wurden. Kompromisse zwischen europäischem und afrikanischem Recht gab es auf folgenden Gebieten, wobei die Aufzählung nur einige Beispiele anführen soll, ohne den Anspruch auf Vollzähligkeit zu erheben:

Im Strafverfahren wurde häufig die Eidesformel nach afrikanischem Recht übernommen; nach europäischem Recht ist der Eid lediglich eine feierliche Erklärung, die Wahrheit zu sagen, der Meineid daher ein die Strafverfolgung nach sich 
ziehendes Verbrechen. Nach afrikanischem Recht dagegen ist der Eid in erster Linie die Anrufung Gottes oder einer Gottheit, so daß ein Falscheid eine Beleidigung der Götter darstellt, die von den übernatürlichen Mächten sanktioniert wird, also keine rechtliche Sanktion erfährt. Da sich die Afrikaner häufig durch die europäische Eidesformel nicht beeindruckt zeigten, wurde die traditionelle Form wieder eingeführt.

Im Eherecht ist häufig den Gatten die Wahl überlassen, vor dem Standesbeamten oder nach einem afrikanischen Brauch die Ehe abzuschließen; bei Wahl der letzten Möglichkeit ist lediglich eine Registrierung der Ehe vor dem Standesamt nachzuholen. Der Brautpreis wurde zum Teil ganz beseitigt, zum Teil der Höhe nach beschränkt oder für gewisse Personengruppen, z. B. Volljährige oder Geschiedene, abgeschafft. Die Polygamie wurde in einigen Fällen beseitigt. Bei der Festlegung der Verwandtschaftsverhältnisse, deren Mitglieder untereinander nicht heiraten dürfen, wurde die nähere Ausgestaltung häufig den Stammesältesten überlassen. Beibehalten wurde in einigen Fällen auch der Brauch der „zeitweiligen Verlassung “. Dieser besagt, daß eine Ehefrau bei Unstimmigkeiten in der Ehe ihren Gatten zeitweilig verlassen kann, um zu ihrem Stamm zurückzukehren. Der Mann kann die Frau nicht an diesem Verhalten hindern noch darf die Ehe innerhalb dieser Zeit aufgelöst werden. Vielmehr ist es üblich, daß er versucht, die Ehefrau durch Geschenke zurückzugewinnen. Schließlich wurde auch die Scheidung nach afrikanischem Recht, d. h. in gegenseitigem Einvernehmen, beibehalten, es wurden aber andererseits auch Scheidungsgründe eingeführt, die nach afrikanischem Recht nicht zur Auflösung der Ehe berechtigen, wie etwa Ehebruch oder Verurteilung eines Gatten zu schweren oder ehrenrührigen Strafen.

Im Grundstücksrecht konnte sich das afrikanische Recht, wenn auch nur teilweise, behaupten. Es ging davon aus, daß der Grund und Boden vom Stamm, von der Familie oder in einigen Fällen von einem besonderen „Bodenherrn" vergeben wurde und daß derjenige, dem Land zugeteilt wurde, dies urbar zu machen und in gutem Zustand zu halten hatte. Solange er dies tat, behielt er den Grund und Boden, wenn er ihn dagegen verließ und er wieder brach lag, fiel er an denjenigen zurück, von dem er ihn erhalten hatte. Das afrikanische Recht ging also in der Frage des Bodenrechts davon aus, daß das Land in erster Linie Kollektiveigentum ist. Damit stand es im Gegensatz zu europäischen Vorstellungen, die Grundbesitz als ein Recht des Individuums ansehen, das ausschließlich und ewig ist, solange sich das besitzende Individuum dieses Rechts nicht begibt, indem es das Grundstück veräußert oder verschenkt. Auf diesem Gebiet fand nun ebenfalls eine Vermischung von europäischen und traditionellen Vorstellungen statt. Um die Jahrhundertwende wurde in allen französischsprachigen Kolonien ein System der Registrierung von Grundstücken eingeführt, weil man erkannt hatte, daß klare und endgültige Grund- und Bodenverhältnisse eine Voraussetzung waren, ein Land wirtschaftlich vernünftig $\mathrm{zu}$ erschließen und $\mathrm{zu}$ entwickeln. Diese Vermischung fand in folgender Weise statt: Immatrikuliert wurde derjenige, der ein bestimmtes Stück Land nach Eingeborenenvorstellungen erhalten hatte, d. h. dem es zur Urbarmachung und Nutzung übergeben worden war. Nach der Registrierung jedoch war das Land unbestrittenes und dauerdes Eigentum desjenigen, $\mathrm{zu}$ dessen Gunsten es eingetragen worden war, selbst wenn dieser Begünstigte aufhörte, das Land weiter zu nutzen. Ahnlich war die Entwicklung in den anderen Kolonialgebieten der übrigen europäischen Mächte. Sie hat sich nach der Unabhängigkeit fortgesetzt und in den Gesetzen verschiedener unabhängiger Staaten ihren Niederschlag gefunden. 


\section{Die Zukunft des afrikanischen Rechts}

Es wäre verfrüht, zum jetzigen Zeitpunkt, d. h., nachdem die meisten afrikanischen Staaten noch keine zehn Jahre lang die Unabhängigkeit besitzen, versuchen zu wollen, endgültige Aussagen über die Zukunft des afrikanischen Rechts und seinen Platz im Gesamtrechtssystem eines afrikanischen Staates zu machen. Eine derartige Beurteilung muß schon aus dem Grunde scheitern, daß bisher noch kein afrikanischer Staat eine Gesamtrevision seiner Gesetze vorgenommen hat, aus der eine eindeutige Stellungnahme zu der Frage, ob er einem der beiden Rechtssysteme den Vorrang einräumen will, herausgelesen werden kann. Aus der bisherigen Tätigkeit der afrikanischen Gesetzgeber geht mit Sicherheit lediglich hervor, daß kein Staat sich für eine der beiden möglichen Radikallösungen entschieden hat, die darin bestünden, entweder das afrikanische Recht oder das europäische Recht zur Gänze abzuschaffen und das jeweils verbleibende Rechtssystem als alleingültig zu übernehmen; eine Ausnahme stellt lediglich Ruanda dar, das das afrikanische Recht abgeschafft hat, dies aber nicht so sehr aus dem Grunde tat, daß es das europäische Recht für besser oder gerechter hält, sondern weil es sein traditionelles Recht als einen Teil des Herrschaftssystems der Watussi ansah, von dem es sich endgültig befreien wollte.

Dennoch dürfte es das traditionelle Recht schwer haben, sich gegen die Einflüsse des europäischen Rechts zu behaupten. Es dürfte deutlich geworden sein, daß das europäische'Recht praktisch für die Regelung aller modernen Lebensbereiche herangezogen wurde, weil sich das afrikanische Recht hierfür als ungeeignet erwies. Es ist zu vermuten, daß das europäische Recht weiterhin diese Rolle spielen wird; dem afrikanischen Recht dürfte allenfalls die Aufgabe zufallen, bei der Regelung spezieller lokal beeinflußter Situationen als Regulativ herangezogen zu werden. Diese Tendenz läßt sich in Ghana erkennen, wo bestimmt ist, daß das allgemeine Recht nicht nur das Recht englischen Ursprungs umfaßt, sondern auch Vorschriften des traditionellen Rechts, die in Gerichtsurteilen anerkannt worden sind. In Somalia hat man bei der Kodifizierung des Zivilrechts neben dem europäischen Recht Elemente des islamischen und des Stammesrechts berücksichtigt. $\mathrm{Zu}$ ähnlichen Ergebnissen versucht man in Tansania zu kommen, indem man auf den Gebieten Bodenrecht, Ehe- und Erbrecht sowie Zivilrecht durch Staatsbeamte das afrikanische Recht sammeln und auf seine Eignung überprüfen läßt, wieweit es zu einem späteren Zeitpunkt mit dem englischen Recht vermischt werden kann. Auch in Äthiopien ist bei der Ausarbeitung moderner Zivil- und Strafgesetze sowie der entsprechenden Prozeßordnungen Gedankengut des traditionellen Rechts übernommen und in die 1960 und 1965 erschienenen Gesetzeswerke eingearbeitet worden.

Abschließend ist noch auf eine besondere Schwierigkeit hinzuweisen, die es meines Erachtens einem Staat in Afrika nahezu unmöglich macht, das afrikanische Recht, selbst wenn er es wollte, gänzlich abzuschaffen. Diese Schwierigkeit besteht in den tatsächlichen Verhältnissen in Afrika, wo infolge des Mangels an Informations- und Kommunikationsmitteln große Teile der Bevölkerung, die weit von den Ballungszentren entfernt wohnen, von Gesetzesreformen gar nicht erreicht werden, sondern weiterhin nach ihren Überlieferungen und Bräuchen leben und mit der modernen Zivilisation nicht in Berührung kommen. Wie soll der Staat hier seine Gesetze durchsetzen? Kann er sich bei Verstößen gegen modernes Recht, von dessen Existenz der betreffende Afrikaner nie gehört hat, auf den allgemein verbreiteten Grundsatz modernen Rechtsdenkens berufen, daß Unwissenheit nicht vor Strafe schützt und den Täter daraufhin verurteilen lassen? Hier wird den afrikanischen Regierungen auf absehbare Zeit nichts anderes übrigbleiben, als die Weiterexistenz afrikanischen Rechts mindestens in den Lebensbereichen der Afrikaner stillschweigend zu dulden, in denen keine besondere und direkte Berührung mit staatlichen Belangen stattfindet. Selbst wenn eine Regierung zur Duldung überkom- 
mener Sitten und Bräuche nicht bereit wäre, bliebe immer noch die Frage, ob sie überhaupt die Autorität hat, ihren Willen im ganzen Land durchzusetzen, oder ob nicht in vielen Fällen ihre Macht an den Grenzen der Hauptstadt und einiger weiterer Zivilisationszentren endet. Antwort auf diese Fragen und damit auch auf die Frage nach der Zukunft des afrikanischen Rechts kann daher nur die Entwicklung der nächsten Jahre oder Jahrzehnte geben.

\section{Wolfgang Heidelberg}

\section{Literaturhinweise}

\section{Sammelwerke}

Afrika Instituut - Studiecentrum

Leiden (Hrsg.)

Baade, Hans W.

Kuper, Hilda und Leo

Poirier, Jean

Einzeldarstellungen

Allott, A. N.

Allot, A. N. (Hrsg.)

Allott, A. N.

Anderson, J. N. D.

Buell, Raymond Leslie

David, René, und Günther Grasmann

Elias, T. Olawale

Elias, T. Olawale

Gonidec, P.-F.

Lord Hailey

Lord Hailey

Jeol, M.

Roberts, Thomas Lee

Rolland, Louis, und Pierre Lampué
The Future of Customary Law in Africa Leiden 1956

African Law: New Law for New Nations New York 1963

African Law: Adaptation and Development Berkeley und Los Angeles 1965

Etudes de Droit Africain et de Droit Malgache

Toulouse 1965

Essays in African Law, with special reference to the law of Ghana London 1960

The Future of Law in Africa: Record of Proceedings of the London Conference 28 December 1959 - 8 January 1960

London 1960

Judicial and Legal Systems in Africa

London 1962

Changing Law in Developing Countries London 1963

The Native Problem in Africa, 2 Bände London und Edinburgh 1965

Einführung in die großen Rechtssysteme der Gegenwart München und Berlin 1966

The Nature of African Customary Law, 2. Aufl.

Manchester 1962

British Colonial Law: A comparative Study of the interaction between English and local Laws in British Dependencies London 1962

Les Droits Africains: Evolution et Sources Paris 1968

An African Survey: A Study of Problems arising in Africa south of the Sahara, 2. Aufl.

London - New York - Toronto 1957

Native Administration in the British African Territor:es, insgesamt 5 Bände, London 1950-1953; davon benutzt:

Bd. I: East Africa: Uganda, Kenya, Tanganyika, 1950

Bd. II: Central Africa: Zansibar, Nyasaland, Northern Rhodesia, 1950

Bd. III: West Africa: Nigeria, Gold Coast, Sierra Leone, Gambia, 1951

Bd. IV: A general survey of the system of native administration, 1951

La Réforme de la Justice en Afrique Noire Paris 1963

Judicial Organisation and Institutions of Contemporary West Africa: a Profile

New York 1966

Droit d'Outre-Mer, 2. Aufl.

Paris 1959 